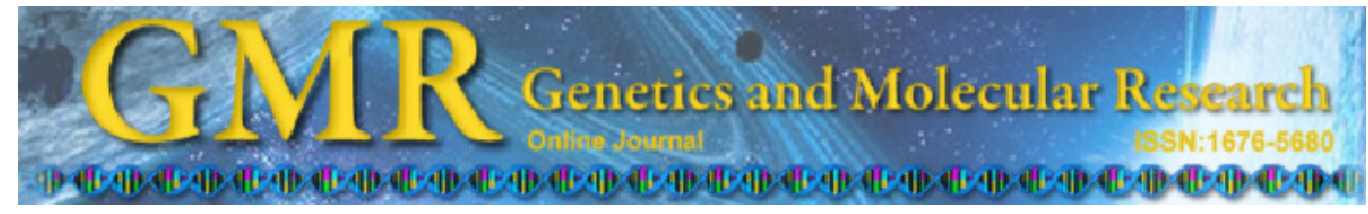

\title{
Novel NKX2-5 mutations responsible for congenital heart disease
}

\author{
J. Wang1*, X.Y. Liu ${ }^{2 *}$ and Y.Q. Yang ${ }^{3 *}$ \\ ${ }^{1}$ Department of Cardiology, East Hospital, \\ Tongji University School of Medicine, Shanghai, China \\ ${ }^{2}$ Department of Pediatrics, Tongji Hospital, \\ Tongji University School of Medicine, Shanghai, China \\ ${ }^{3}$ Department of Cardiovascular Research, Shanghai Chest Hospital, \\ Medical College of Shanghai Jiaotong University, Shanghai, China \\ *These authors contributed equally to this study. \\ Corresponding author: Y.Q. Yang \\ E-mail: yang99yang66@hotmail.com
}

Genet. Mol. Res. 10 (4): 2905-2915 (2011)

Received December 3, 2010

Accepted May 5, 2011

Published November 29, 2011

DOI http://dx.doi.org/10.4238/2011.November.29.1

\begin{abstract}
Congenital heart disease (CHD) is the most common birth defect and is the leading cause of infant morbidity and mortality resulting from birth defects. Increasing evidence demonstrates that genetic variation in the $N K X 2-5$ gene, which encodes a homeobox-containing transcription factor crucial to cardiogenesis, is an important molecular determinant for CHD. Nevertheless, the genetic components underlying CHD remain largely unknown. We screened $N K X 2-5$ for potential molecular defects in patients with $\mathrm{CHD}$. The entire coding region of NKX2-5 was initially sequenced in a cohort of 268 unrelated patients with CHD. The relatives of the patients carrying identified mutations and 200 unrelated control individuals were subsequently genotyped. Three novel heterozygous missense $N K X 2-5$ mutations, p.Q22K, p.R36S, and p.E54K, were identified in three families with autosomal dominantly inherited atrial septal defect, ventricular septal defect, and tetralogy of Fallot, respectively. These mutations, absent in 200 control individuals, appear to be highly conserved evolutionarily and co-segregated with
\end{abstract}


CHD in the families, with complete penetrance. These findings expand the spectrum of mutations in NKX2-5 associated with CHD and provide new insight into the molecular etiology involved in the pathogenesis of CHD, which signifies potential implications for genetic diagnosis and gene-specific therapy for this common disease in newborns.

Key words: Congenital heart disease; Transcription factor; Genetics

\section{INTRODUCTION}

Congenital heart disease (CHD) is the most prevalent form of birth defect in the structure of the heart or intrathoracic great vessels of a neonate, affecting nearly $1 \%$ of newborns, and is the most common cause of infant death resulting from birth abnormality, with more than $29 \%$ deaths of infants with a heart birth defect (Lloyd-Jones et al., 2009). Despite its striking prevalence and clinical significance, the etiology of CHD in the majority of cases is unclear (Jenkins et al., 2007; Pierpont et al., 2007). Abnormal cardiac development during embryogenesis seems to occur through a biological process that is heterogeneous and complex, with both environmental and genetic risk factors (Nora and Nora, 1976). Based on molecular genetic studies in CHD pedigrees with Mendelian inheritance patterns, several genes have been identified to be involved in the pathogenesis of CHD, most of which encode cardiac transcription factors such as NKX2-5, TBX5, GATA4, and GATA6 (Li et al., 1997; Schott et al., 1998; Garg et al., 2003; Kodo et al., 2009).

NKX2-5 is the earliest known marker of myocardial progenitor cells in all species in which it has been studied (Akazawa and Komuro, 2005). The human NKX2-5 gene maps to chromosome $5 \mathrm{q} 34$ and consists of two exons encoding a protein of 324 amino acids (Shiojima et al., 1995). This homeobox transcription factor is expressed during early cardiac morphogenesis and functions as a pivotal regulatory protein (Akazawa and Komuro, 2005). Due to its crucial role in normal cardiogenesis, $N K X 2-5$ has been a prime candidate gene in studies to identify the genetic determinants for congenital structural heart defects. To date, more than 40 mutations within the NKX2-5 gene have been identified in patients with a variety of congenital heart malformations, including atrial septal defect with normal or abnormal atrioventricular conduction, ventricular septal defect, conotruncal abnormalities as tetralogy of Fallot, double-outlet right ventricle, L-transposition of the great arteries, and hypoplastic left heart syndrome (Stallmeyer et al., 2010). These observations strongly suggest that NKX2-5 is important in the later stages of heart development and maturation, in addition to its role in cardiac progenitor commitment and patterning in the developing heart (Prall et al., 2007).

To identify the molecular etiology responsible for CHD, a systematic scan of the NKX2-5 gene for likely mutations was performed by sequencing it initially in a cohort of 268 unrelated patients with various types of CHD and subsequently in the family members of the patients harboring mutations, in contrast to a total of 200 unrelated control individuals.

\section{MATERIAL AND METHODS}

\section{Study subjects}

A cohort of 268 unrelated patients with CHD, who underwent surgical repair or percutaneous closure at hospitals from January 2006 to May 2010, was recruited prospec- 
tively. They were clinically evaluated by cardiologists for their individual and familial history, review of the medical records, complete physical examination, standard 12-lead electrocardiogram (ECG), and two-dimensional transthoracic echocardiography with color flow Doppler. Transesophageal echocardiography, cardiac catheterization, electrophysiological investigation, and/or cardiac surgery had previously been conducted in some individuals. Patients with syndromic CHD, such as Marfan, Noonan, Holt-Oram, Alagille, and CHARGE syndromes, as well as chromosomal abnormalities that are highly associated with CHD, such as trisomy 21 (Down syndrome) and 22q11.2 deletion (DiGeorge syndrome), were excluded from the present study. Family members of mutation carriers were subsequently recruited, and their medical records evaluated. We also carried out a physical examination, ECG, and echocardiography, and all individuals were screened for the identified mutations in NKX2-5 by direct sequencing. A total of 200 unrelated, ethnically matched healthy individuals, who were enrolled from the general population and had no evidence for any type of CHD following the comprehensive evaluation of their individual and familial histories, detailed review of the medical records, and complete physical examination (Stallmeyer et al., 2010), were used as controls to screen for the identified mutations. Peripheral venous blood samples were collected from subjects and control individuals. The study protocol was reviewed and approved by the local institutional Ethics Committee and written informed consent was obtained from all participants or their guardians prior to the study.

\section{Genetic studies}

Genomic DNA from all participants was extracted from blood lymphocytes with the Wizard Genomic DNA Purification Kit (Promega, Madison, WI, USA). First, the candidate gene $N K X 2-5$ was screened in 268 unrelated patients with CHD. Then, the genotyping NKX2-5 in the available relatives of the patients carrying the identified mutations and the 200 unrelated, ethnically matched healthy control individuals was carried out subsequently for the presence of mutations identified in the patients. The referential genomic DNA sequence of NKX2-5 was derived from GenBank (accession No. NT 023133). With the help of the Primer3 software (http://frodo.wi.mit.edu), the primer pairs used to amplify the complete coding region of $N K X 2-5$ by polymerase chain reaction (PCR) were designed as follows: primer 1 forward 5'-CAC GAT GCA GGG AAG CTG-3', and reverse 5'-AGT TTC TTG GGG ACG AAA GC-3' (the PCR product was $477 \mathrm{bp}$ in size); primer 2 forward 5'-CCT CCA CGA GGA TCC CTT AC-3', and reverse 5'-CGA GTC CCC TAG GCA TGG-3' (the product was $463 \mathrm{bp}$ ); primer 3 forward 5'-AGA ACC GGC GCT ACA AGT G-3', and reverse 5'-GAG TCA GGG AGC TGT TGA GG-3' (the product was 473 bp). PCR was performed using HotStar Taq DNA Polymerase (Qiagen, Hilden, Germany) on a PE 9700 Thermal Cycler (Applied Biosystems, Foster, CA, USA), under standard conditions and concentrations of reagents. Amplified products were analyzed on 1\% agarose gels stained with ethidium bromide and purified with QIAquick Gel Extraction Kit (Qiagen). Both strands of each PCR product were sequenced with a BigDye ${ }^{\circledR}$ Terminator v3.1 Cycle Sequencing Kit (Applied Biosystems) under an ABI PRISM 3130 XL DNA Analyzer (Applied Biosystems). The sequencing primers used were previously designed for specific region sequencing. The DNA sequences were viewed and analyzed with the 
DNA Sequencing Analysis Software v5.1 (Applied Biosystems). The variant was validated by re-sequencing an independent PCR-generated amplicon from the subject and met our quality control thresholds with a call rate $>99 \%$.

\section{Multiple sequence alignments}

The multiple NKX2-5 protein sequences across various species were aligned using the MUSCLE program (version 3.6, URL: http://www.ncbi.nlm.nih.gov/sites/entrez?cmd=Re trieve $\& \mathrm{db}=$ homologene $\&$ dopt $=$ MultipleAlignment\&list_uids=3230).

\section{Prediction of the disease-causing potential of a sequence alteration}

The disease-causing potential of a sequence alteration detected in NKX2-5 was predicted automatically by the MutationTaster program (http://www.mutationtaster.org/), suggesting that the alteration could either be a disease mutation or a harmless polymorphism. Notably, the $\mathrm{P}$ value used here is the prediction probability rather than the probability of error as used in Student $t$-test statistics, i.e., a value close to 1 indicates a high prediction probability.

\section{RESULTS}

\section{Characteristics of the study subjects}

A cohort of 268 unrelated patients with CHD, of which 53 patients had a positive family history, was recruited and clinically evaluated, in contrast to a total of 200 unrelated, ethnically matched healthy individuals as controls. All subjects were of Chinese Han nationality. None of them had overt environmental risk factors for CHD. The clinical characteristics of the 268 unrelated patients with CHD are shown in Table 1.

\section{NKX2-5 mutations}

Direct sequencing of the coding exons of the $N K X 2-5$ gene was performed after PCR amplification of genomic DNA from a cohort of 268 unrelated patients with CHD. Three novel heterozygous mutations in NKX2-5 were identified in 3 of 268 unrelated patients. The total prevalence of $N K X 2-5$ mutations based on the cohort population was approximately $1.12 \%$. A substitution of adenine (A) for cytosine (C) in the first nucleotide of codon 22 (alternatively c.64C $>\mathrm{A}$ ) of the $N K X 2-5$ gene, corresponding to the transversion of glutamine (Q) to lysine (K) at the amino acid position 22 (p.Q22K), was identified in a patient with an atrial septal defect. A transversion of CGC to AGC at codon 36 (c. $106 \mathrm{C}>\mathrm{A}$ ) of the NKX2-5 gene, predicting the change from arginine $(\mathrm{R})$ to serine $(\mathrm{S})$ at the amino acid position 36 (p.R36S), was identified in a patient with ventricular septal defect. A transition of guanine (G) to $A$ at the nucleotide position 160 from the translation starting point (c.160G $>A$ ) of the NKX2-5 gene, equivalent to the change of the encoded amino acid from glutamic acid (E) to K (p.E54K), was identified in a patient with tetralogy of Fallot. The prevalence of these mutations in patients with atrial septal defect, ventricular septal defect, or tetralogy of Fallot was approximately $2.59 \%$. The sequence chromatograms showing the detected heterozy- 


\begin{tabular}{|c|c|c|}
\hline & Number or mean value & Percentage or range \\
\hline Male:female & 152:116 & $57: 43$ \\
\hline Age at the present study (years) & 5.62 & $0.25-38$ \\
\hline Age at the diagnosis of CHD (years) & 3.74 & $0-16$ \\
\hline Positive family history & 53 & 19.78 \\
\hline \multicolumn{3}{|l|}{ Distribution of different types of CHD } \\
\hline Isolated CHD & 211 & 78.73 \\
\hline ASD & 105 & 39.18 \\
\hline VSD & 86 & 32.09 \\
\hline PDA & 5 & 1.87 \\
\hline DORV & 3 & 1.12 \\
\hline PS & 3 & 1.12 \\
\hline TAPVC & 3 & 1.12 \\
\hline $\mathrm{COA}$ & 2 & 0.75 \\
\hline TGA & 2 & 0.75 \\
\hline CAVC & 1 & 0.37 \\
\hline Cor triatriatum & 1 & 0.37 \\
\hline Complex CHD & 57 & 21.27 \\
\hline ASD+VSD & 17 & 6.34 \\
\hline TOF & 15 & 5.60 \\
\hline VSD+PDA & 6 & 2.24 \\
\hline VSD+PFO & 5 & 1.87 \\
\hline VSD+DORV & 4 & 1.49 \\
\hline VSD+TGA & 3 & 1.12 \\
\hline $\mathrm{VSD}+\mathrm{PFO}+\mathrm{PDA}$ & 2 & 0.75 \\
\hline $\mathrm{ASD}+\mathrm{PDA}$ & 1 & 0.37 \\
\hline ASD+TGA & 1 & 0.37 \\
\hline $\mathrm{ASD}+\mathrm{VSD}+\mathrm{DORV}$ & 1 & 0.37 \\
\hline ASD+VSD+PDA & 1 & 0.37 \\
\hline $\mathrm{COA}+\mathrm{PDA}$ & 1 & 0.37 \\
\hline \multicolumn{3}{|l|}{ Incidence of arrhythmias } \\
\hline Atrioventricular block & 12 & 4.48 \\
\hline Atrial fibrillation & 3 & 1.12 \\
\hline \multicolumn{3}{|l|}{ Treatment } \\
\hline Surgical repair & 165 & 61.57 \\
\hline Percutaneous closure & 78 & 29.10 \\
\hline
\end{tabular}

gous $N K X 2-5$ variations of c. $64 \mathrm{C}>\mathrm{A}, \mathrm{c} .106 \mathrm{C}>\mathrm{A}$, and $\mathrm{c} .160 \mathrm{G}>\mathrm{A}$ in comparison to control sequences are shown in Figure 1. These three variants were not present in the 200 unrelated control individuals. Genetic scan of the family members of the three unrelated patients harboring the identified mutations showed that the gene variant was present in all affected family members alive, but absent in unaffected family members tested in each family. Analysis of the pedigrees demonstrated that the mutation co-segregated with CHD was transmitted as an autosomal dominant trait in the three families with complete penetrance. The pedigree structures of the three families are illustrated in Figure 2. The phenotypic characteristics and results of genetic screening of the affected pedigree members are summarized in Table 2.

\section{Multiple alignments of the NKX2-5 protein sequences across species}

A cross-species alignment of NKX2-5 protein sequences showed that the altered amino acids were highly conserved evolutionarily as shown in Figure 3, suggesting that these amino acids are functionally important. 


\section{A}
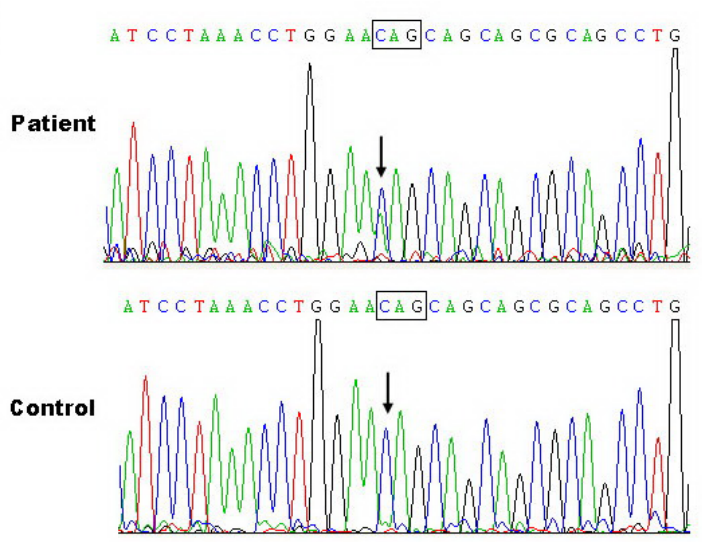

B
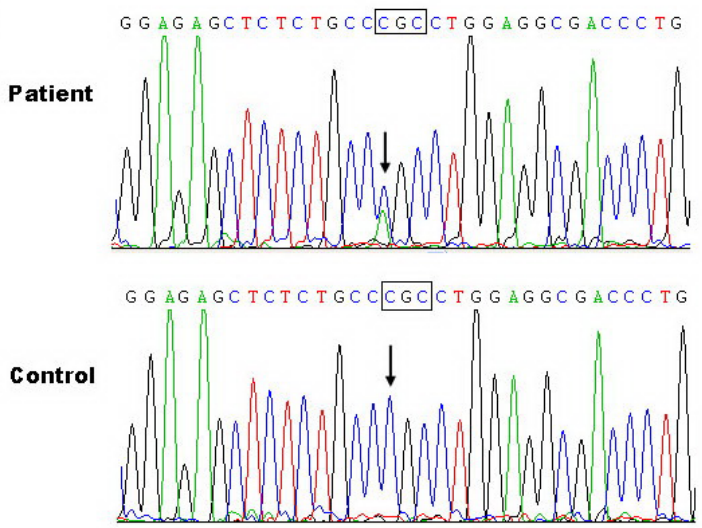

C
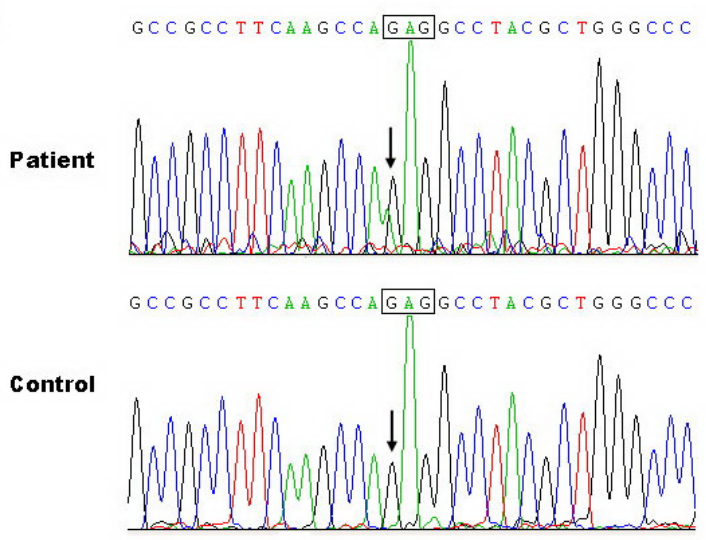

Figure 1. Sequence chromatograms of NKX2-5 in index patients and controls. Arrows indicate the heterozygous nucleotides of $\mathrm{C} / \mathrm{A}(\mathbf{A}), \mathrm{C} / \mathrm{A}(\mathbf{B})$ and $\mathrm{G} / \mathrm{A}(\mathbf{C})$, in the probands from families 1,2 and 3 (Patient), respectively, or the homozygous nucleotides of $\mathrm{C} / \mathrm{C}(\mathbf{A}), \mathrm{C} / \mathrm{C}(\mathbf{B})$ and $\mathrm{G} / \mathrm{G}(\mathbf{C})$ in the corresponding controls (Control). Squares denote the nucleotides comprising a codon of NKX2-5. 




III-1

\section{Family 1}

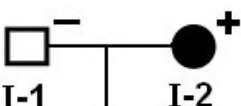

$\mathrm{I}-1$

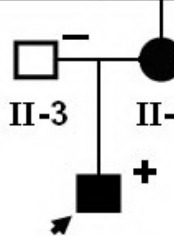

III-2
Family 2



Family 3



Figure 2. Pedigree structures of families with atrial septal defects. Families are designated as family 1, 2, and 3. Family members are identified by generations and numbers. Squares $=$ males; circles $=$ females; closed symbols $=$ affected members; open symbols $=$ unaffected members; arrows $=$ proband $;+=$ carriers of the heterozygous mutations; - = non-carriers.

Table 2. Phenotypic characteristics and status of NKX2-5 mutations of the affected pedigree members.

\begin{tabular}{|c|c|c|c|c|c|c|c|}
\hline Identity & Gender & $\begin{array}{l}\text { Age at time of } \\
\text { study (years) }\end{array}$ & $\begin{array}{c}\text { Age at diagnosis of } \\
\text { CHD (years) }\end{array}$ & $\begin{array}{c}\text { Cardiac structural } \\
\text { defects }\end{array}$ & $\begin{array}{c}\text { Other structural } \\
\text { abnormalities }\end{array}$ & Arrhythmias & $\begin{array}{c}N K X 2-5 \\
\text { mutations }\end{array}$ \\
\hline Family 1 & & & & & & & Q22K \\
\hline $\mathrm{I}-2$ & $\mathrm{~F}$ & 59 & 23 & ASD & PS, RVH & Second-degree AVB & $+/-$ \\
\hline II-1 & M & 36 & 19 & ASD & - & First-degree AVB & $+/-$ \\
\hline II-4 & $\mathrm{F}$ & 32 & 15 & ASD & - & First-degree AVB & $+/-$ \\
\hline II-6 & $\mathrm{F}$ & 29 & 12 & ASD & - & - & $+/-$ \\
\hline III-2 & $\mathrm{M}$ & 5 & 5 & ASD & & - & $+/-$ \\
\hline Family 2 & & & & & & & R36S \\
\hline $\mathrm{I}-1$ & M & 30 & 9 & VSD & - & Paroxysmal AF & $+/-$ \\
\hline II-1 & M & 6 & 3 & VSD & - & - & $+/-$ \\
\hline Family 3 & & & & & & & E54K \\
\hline $\mathrm{I}-2$ & $\mathrm{~F}$ & 57 & 9 & TOF & BPV & - & $+/-$ \\
\hline II-1 & $\mathrm{M}$ & 22 & 2 & TOF & - & - & $+/-$ \\
\hline II-2 & $\mathrm{F}$ & 19 & 1 & TOF & - & - & $+/-$ \\
\hline
\end{tabular}

$\mathrm{F}=$ female $; \mathrm{M}=$ male $; \mathrm{CHD}=$ congenital heart disease $; \mathrm{ASD}=$ atrial septal defect $; \mathrm{VSD}=$ ventricular septal defect $;$ TOF $=$ tetralogy of Fallot; PS = pulmonary stenosis; RVH = right ventricular hypertrophy; $\mathrm{BPV}=$ bicuspid pulmonary valve; $\mathrm{AVB}=$ atrioventricular block; $+=$ present; $-=$ absent.

\begin{tabular}{|c|c|c|c|c|}
\hline & 10 & $22 \mathrm{~K}$ & $6 \mathrm{~S}$ & $4 K$ \\
\hline Human & --- TPFSVKDILNLE & QQRRSLAAAGELSA R & LEATLAP - SSCMLAAF KP & AYAGPEAAAP -- \\
\hline Chimpanzee & -- - TPFSVKDILNLE & Q QQRSLAAAGELSA R & LEATLAP - SSCMLAAF KP & AYAGPEAAAP \\
\hline & -- - TPFSVKDILNLE & Q QQRSL - AAGELSAR & LEATLAP - ASCMLAAF KP & AYAGPEAAAP --- \\
\hline Cattle & --- TPFSVKDILNLE & Q QQRSL - AAGELSA R & LEATLAP - ASCMLAAF KP & AYAGPEAAAP \\
\hline Mouse & - TPFSVKDILNLE & Q QQRSL - AS G DLSA R & LEATLAP - ASCMLAAF KP & AYSGPEAAAS - - \\
\hline & -- - TPFSVKDILNLE & Q QQRSL - AAG DLSA R & LEATLAP - ASCMLAAF KP & GYSGPEAAAP - - \\
\hline $\begin{array}{l}\text { Fowl } \\
\text { Zebrafish }\end{array}$ & $\begin{array}{l}\text {-- TPFSVKDILNLE } \\
--- \text { TPFSYRDILNLE }\end{array}$ & $\begin{array}{l}\text { Q QQQGGLAPMELSS } \\
\text { O NOEDM-VS LDSO R }\end{array}$ & - . PSCMLATFKQ & AFG - . SEPP \\
\hline
\end{tabular}

Figure 3. Alignment of multiple NKX2-5 protein sequences across species. The altered amino acids of Q22, R36, and E54 are highly conserved evolutionarily. 


\section{Disease-causing potential of a sequence alteration}

The sequence alterations of c. $64 \mathrm{C}>\mathrm{A}, \mathrm{c} .106 \mathrm{C}>\mathrm{A}$, and c. $160 \mathrm{G}>\mathrm{A}$ detected in $N K X 2-5$ were all predicted to be disease-causing, with $\mathrm{P}$ values of $0.99743,0.99098$, and 0.99769 for c. $64 \mathrm{C}>\mathrm{A}, \mathrm{c} .106 \mathrm{C}>\mathrm{A}$, and c. $160 \mathrm{G}>\mathrm{A}$, respectively, providing evidence for the three alterations to be malicious disease mutations rather than benign polymorphisms.

\section{DISCUSSION}

In the present study, we report three previously unrecognized missense mutations of NKX2-5 identified in patients with familial CHD. These novel heterozygous mutations were present in all the affected family members alive but absent in unaffected relatives tested and 400 normal chromosomes from a matched control population. A cross-species alignment of NKX2-5 protein sequences showed that the altered amino acids were highly conserved evolutionarily. Prediction of causative potential of a sequence alteration demonstrated that all three mutations of p.Q22K, p.R36S, and p.E54K were disease-causing with probability values as high as close to 1 . Therefore, it is very likely that the three mutations are associated with the CHD in these three families.

Our results are supported by the finding of other $N K X 2-5$ mutations predisposing to congenital CHD by impairing or reducing cardiac transcription factor NKX2-5 (Kasahara et al., 2000; Kasahara and Benson, 2004). To date, more than 40 mutations in NKX2-5 have been described, of which more than 30 mutations have been observed in patients with atrial septal defect, showing that although $N K X 2-5$ mutations are involved in a long list of cardiac malformations, the most frequent phenotype resulted is atrial septal defect (Stallmeyer et al., 2010). In most of these patients, the CHD-causing mutations are familial, whereas sporadic cases remain relatively infrequent (Elliott et al., 20003; Sarkozy et al., 2005). Similar to these findings, the figure of $3 / 53$ mutations (approximately $6 \%$ ) in our patient cohort with positive family history suggests that the NKX2-5 mutations could be a major cause of familial CHD. Notably, the remarkable genetic heterogeneity of CHD was proven by an inability to detect mutations in nearly $99 \%$ of our cohort patients, despite somatic NKX2-5 mutations being a likely mechanism of CHD in some patients (Reamon-Buettner and Borlak, 2004). Hence, the contribution of genes other than NKX2-5 to CHD pathogenesis seems probable.

Mutations in other transcription factors associated with cardiogenesis, such as TBX5, GATA4, GATA6, and NOTCH1 (Li et al., 1997; Garg et al., 2003, 2005; Kodo et al., 2009), and mutations in genes, such as RAF1, SOS1 and BRAF within signal transduction pathways (Rodriguez-Viciana et al., 2006; Razzaque et al., 2007; Roberts et al., 2007), have also been detected in patients with CHD. Also, mutations in cardiac structural proteins as alpha myosin heavy chain (MYH6) and alpha cardiac actin (ACTC1) were identified in familial CHD (Ching et al., 2005; Matsson et al., 2008). However, so far, only NKX2-5 mutations were reported to cause an atrial septal defect phenotype and development of atrioventricular block (Stallmeyer et al., 2010). The two most common phenotypes caused by mutated NKX2-5 are atrial septal defect and atrioventricular conduction disturbance (Akazawa and Komuro, 2005), indicating the crucial role of $N K X 2-5$ not only in the morphogenesis of the heart, but also in the construction of cardiac conduction system. In the present study, a compound phenotype of atrial septal defect and atrioventricular block was observed in three fifths of the members of family 1 who carried the identified mutations of NKX2-5, and the atrioventricular block seemed to be 
progressive with increasing age in each individual as described previously (Schott et al., 1998; Hosoda et al., 1999; Pashmforoush et al., 2004; Hirayama-Yamada et al., 2005; Sarkozy et al., 2005), while it is not observed soon after birth. As atrioventricular block is a possible cause of sudden death, molecular genetic screening appears to be of great help in identifying individuals at risk for the life-threatening heart conduction disturbances. When a person harboring an NKX2-5 mutation is identified, it is necessary for medical staff to carefully monitor this patient who initially may present no symptoms, or may have spontaneously closed or surgically corrected atrial septal defect, which enables offering accurate and early therapy.

Association of compromised NKX2-5 with increased predisposition to CHD and atrioventricular block has been reported in animal models. The homeobox-containing transcription factor encoded by the tinman gene, a counterpart of $N K X 2-5$, was expressed in the dorsal vessel (an equivalent to the heart of vertebrates) of the fruit fly Drosophila melanogaster and the deletion of the transcription factor led to lethal failure of vessel formation (Benson et al., 1999). In Xenopus, expression of a similar DNA-nonbinding mutant of $\mathrm{Nkx} 2.5$ was demonstrated to cause dominant negative effect on embryos, showing small heart or no heart formation (Grow and Krieg, 1998). In mice, $N K X 2-5$ was highly expressed in the early heart progenitor cells in both primary and secondary heart fields during embryogenesis and continued to be expressed at a high level in the heart through adulthood. In particular, a transiently elevated expression of NKX2-5 was observed in specialized myocardial conduction cells during the development of cardiac conduction system (Akazawa and Komuro, 2005). In transgenic mice expressing a DNA binding-impaired mutant of mouse NKX2-5 (I183P), under the $\beta$-myosin heavy chain promoter, the accumulation of mutant protein in the embryo, neonate, and adult myocardium resulted in progressive and profound cardiac conduction defects and heart failure (Kasahara et al., 2001). Targeted disruption of NKX2-5 in mice caused embryonic lethality around ED10.5, with retarded cardiac development (Lyons et al., 1995; Tanaka et al., 1999). Mice heterozygous for NKX2-5null alleles were predisposed to atrial septal defect and abnormal atrioventricular conduction (Biben et al., 2000). Ventricular-restricted NKX2-5 knockout around ED8.0 to ED8.5, created by crossing floxed-NKX2-5 mice with myosin light chain $2 \mathrm{v}$-Cre knock-in mice, gave rise to progressive and advanced conduction defects and left ventricular hypertrophy postnatally (Pashmforoush et al., 2004). In addition, perinatal loss of Nkx2-5 brought about rapid conduction and contraction defects by regulating expression of several ion channel genes (Briggs et al., 2008). Taken together, these experimental findings in animals suggest that $N K X 2-5$ mutations underlie a variety of congenital cardiac abnormalities in humans, including atrial septal defect with or without progressive conduction anomaly, ventricular septal defect, and tetralogy of Fallot.

\section{CONCLUSIONS}

The findings of the present study link novel mutations in the cardiac transcription factor NKX2-5 to CHD and provide a new insight into the molecular mechanism implicated in the pathogenesis of CHD, representing an important step in progress toward molecular phenotyping and thus directed rather than empiric therapy for this common disease in newborns.

\section{ACKNOWLEDGMENTS}

We are indebted to participants for their dedication to the study. Research supported 
in part by grants from the Natural Science Fund of Shanghai, China (\#10ZR1433100), the National Natural Science Fund of China (\#30570768, \#81070153 and \#30700776), and the National Basic Research Program of China (\#2010CB912604).

\section{REFERENCES}

Akazawa H and Komuro I (2005). Cardiac transcription factor Csx/Nkx2-5: its role in cardiac development and diseases. Pharmacol. Ther. 107: 252-268.

Benson DW, Silberbach GM, Kavanaugh-McHugh A, Cottrill C, et al. (1999). Mutations in the cardiac transcription factor NKX2.5 affect diverse cardiac developmental pathways. J. Clin. Invest. 104: 1567-1573.

Biben C, Weber R, Kesteven S, Stanley E, et al. (2000). Cardiac septal and valvular dysmorphogenesis in mice heterozygous for mutations in the homeobox gene Nkx2-5. Circ. Res. 87: 888-895.

Briggs LE, Takeda M, Cuadra AE, Wakimoto H, et al. (2008). Perinatal loss of Nkx2-5 results in rapid conduction and contraction defects. Circ. Res. 103: 580-590.

Ching YH, Ghosh TK, Cross SJ, Packham EA, et al. (2005). Mutation in myosin heavy chain 6 causes atrial septal defect. Nat. Genet. 37: 423-428.

Elliott DA, Kirk EP, Yeoh T, Chandar S, et al. (2003). Cardiac homeobox gene NKX2-5 mutations and congenital heart disease: associations with atrial septal defect and hypoplastic left heart syndrome. J. Am. Coll. Cardiol. 41: 2072-2076.

Garg V, Kathiriya IS, Barnes R, Schluterman MK, et al. (2003). GATA4 mutations cause human congenital heart defects and reveal an interaction with TBX5. Nature 424: 443-447.

Garg V, Muth AN, Ransom JF, Schluterman MK, et al. (2005). Mutations in NOTCH1 cause aortic valve disease. Nature 437: $270-274$.

Grow MW and Krieg PA (1998). Tinman function is essential for vertebrate heart development: elimination of cardiac differentiation by dominant inhibitory mutants of the tinman-related genes, XNkx2-3 and XNkx2-5. Dev. Biol. 204: 187-196.

Hirayama-Yamada K, Kamisago M, Akimoto K, Aotsuka H, et al. (2005). Phenotypes with GATA4 or NKX2.5 mutations in familial atrial septal defect. Am. J. Med. Genet. A 135: 47-52.

Hosoda T, Komuro I, Shiojima I, Hiroi Y, et al. (1999). Familial atrial septal defect and atrioventricular conduction disturbance associated with a point mutation in the cardiac homeobox gene CSX/NKX2-5 in a Japanese patient. Jpn. Circ. J. 63: 425-426.

Jenkins KJ, Correa A, Feinstein JA, Botto L, et al. (2007). Noninherited risk factors and congenital cardiovascular defects: current knowledge: a scientific statement from the American Heart Association Council on Cardiovascular Disease in the Young: endorsed by the American Academy of Pediatrics. Circulation 115: 2995-3014.

Kasahara H and Benson DW (2004). Biochemical analyses of eight NKX2.5 homeodomain missense mutations causing atrioventricular block and cardiac anomalies. Cardiovasc. Res. 64: 40-51.

Kasahara H, Lee B, Schott JJ, Benson DW, et al. (2000). Loss of function and inhibitory effects of human CSX/NKX2.5 homeoprotein mutations associated with congenital heart disease. J. Clin. Invest. 106: 299-308.

Kasahara H, Wakimoto H, Liu M, Maguire CT, et al. (2001). Progressive atrioventricular conduction defects and heart failure in mice expressing a mutant Csx/Nkx2.5 homeoprotein. J. Clin. Invest. 108: 189-201.

Kodo K, Nishizawa T, Furutani M, Arai S, et al. (2009). GATA6 mutations cause human cardiac outflow tract defects by disrupting semaphorin-plexin signaling. Proc Natl. Acad. Sci U. S. A. 106: 13933-13938.

Li QY, Newbury-Ecob RA, Terrett JA, Wilson DI, et al. (1997). Holt-Oram syndrome is caused by mutations in TBX5, a member of the Brachyury (T) gene family. Nat. Genet. 15: 21-29.

Lloyd-Jones D, Adams R, Carnethon M, De Simone G, et al. (2009). Heart disease and stroke statistics--2009 update: a report from the American Heart Association Statistics Committee and Stroke Statistics Subcommittee. Circulation 119: e21-181.

Lyons I, Parsons LM, Hartley L, Li R, et al. (1995). Myogenic and morphogenetic defects in the heart tubes of murine embryos lacking the homeo box gene Nkx2-5. Genes Dev. 9: 1654-1666.

Matsson H, Eason J, Bookwalter CS, Klar J, et al. (2008). Alpha-cardiac actin mutations produce atrial septal defects. Hum. Mol. Genet. 17: 256-265.

Nora JJ and Nora AH (1976). Recurrence risks in children having one parent with a congenital heart disease. Circulation 53: 701-702.

Pashmforoush M, Lu JT, Chen H, Amand TS, et al. (2004). Nkx2-5 pathways and congenital heart disease; loss of ventricular myocyte lineage specification leads to progressive cardiomyopathy and complete heart block. Cell 117: 373-386.

Genetics and Molecular Research 10 (4): 2905-2915 (2011)

CFUNPEC-RP www.funpecrp.com.br 
Pierpont ME, Basson CT, Benson DW Jr, Gelb BD, et al. (2007). Genetic basis for congenital heart defects: current knowledge: a scientific statement from the American Heart Association Congenital Cardiac Defects Committee, Council on Cardiovascular Disease in the Young: endorsed by the American Academy of Pediatrics. Circulation 115: 3015-3038.

Prall OW, Menon MK, Solloway MJ, Watanabe Y, et al. (2007). An Nkx2-5/Bmp2/Smad1 negative feedback loop controls heart progenitor specification and proliferation. Cell 128: 947-959.

Razzaque MA, Nishizawa T, Komoike Y, Yagi H, et al. (2007). Germline gain-of-function mutations in RAF1 cause Noonan syndrome. Nat. Genet. 39: 1013-1017.

Reamon-Buettner SM and Borlak J (2004). Somatic NKX2-5 mutations as a novel mechanism of disease in complex congenital heart disease. J. Med. Genet. 41: 684-690.

Roberts AE, Araki T, Swanson KD, Montgomery KT, et al. (2007). Germline gain-of-function mutations in SOS1 cause Noonan syndrome. Nat. Genet. 39: 70-74.

Rodriguez-Viciana P, Tetsu O, Tidyman WE, Estep AL, et al. (2006). Germline mutations in genes within the MAPK pathway cause cardio-facio-cutaneous syndrome. Science 311: 1287-1290.

Sarkozy A, Conti E, Neri C, D'Agostino R, et al. (2005). Spectrum of atrial septal defects associated with mutations of NKX2.5 and GATA4 transcription factors. J. Med. Genet. 42: e16.

Schott JJ, Benson DW, Basson CT, Pease W, et al. (1998). Congenital heart disease caused by mutations in the transcription factor NKX2-5. Science 281: 108-111.

Shiojima I, Komuro I, Inazawa J, Nakahori Y, et al. (1995). Assignment of cardiac homeobox gene CSX to human chromosome 5q34. Genomics 27: 204-206.

Stallmeyer B, Fenge H, Nowak-Gottl U and Schulze-Bahr E (2010). Mutational spectrum in the cardiac transcription factor gene NKX2.5 (CSX) associated with congenital heart disease. Clin. Genet. 78: 533-540.

Tanaka M, Chen Z, Bartunkova S, Yamasaki N, et al. (1999). The cardiac homeobox gene Csx/Nkx2.5 lies genetically upstream of multiple genes essential for heart development. Development 126: 1269-1280. 